\title{
Stereopsis from reversible and irreversible patterns
}

\author{
R. B. LAWSON, SARA PACKARD, DAWN LAWRENCE, and C. L. G. WHITMORE \\ University of Vermont, Burlington, Vermont 05401
}

\begin{abstract}
Eight subjects reported stereoscopic depth as a function of the magnitude and direction of disparity carried by a reversible grid or an irreversible solid surface. The former alternated between a diamond seen against a grating or a uniform rectangular grid pattern with stereoscopic depth reported only when the diamond was perceived. Depth was attenuated for the grid compared to the solid surface patterns. Disparity can be defined by perceptually extracted forms with depth present only when those forms are bounded by visible contours.
\end{abstract}

The importance of disparity as the stimulus for stereoscopic depth perception has long been recognized, even though the precise definition of disparity continues to attract the attention of visual scientists (Gulick \& Lawson, 1976; Julesz, 1971; Pastore, 1972; Wallach \& Lindauer, 1962; Wallach \& Bacon, 1976). Obviously, the lateral displacement of a portion of the monocular views is critical to generating stereoscopic depth regardless of whether monocular contours (Ogle, 1959), submatrices of random elements (Julesz, 1971; Julesz \& Frisby, 1975), homogeneous regions (Lawson \& Gulick, 1967), or superimposed brightness patterns (Kaufman \& Pitblado, 1965) are shifted. These findings indicate that the binocular visual system can detect global as well as local transverse displacements that can be carried by a variety of stimulus properties apart from identifiable monocular contours (Gulick \& Lawson, 1976; Nelson, 1975).

Common to all of the above stereoscopic displays is not only the presence of a displaced portion of the half-images but the fact that the perceptual organization of the monocular patterns is quite stable. For example, when viewing a half-image from a random-element stereogram, one sees only a randomly textured surface of dots (Julesz, 1971) or lines (Julesz \& Frisby, 1975) without any periodic monocular recognition of the displaced portion of the matrix, which only becomes visible and perceptually stabilized following binocular combination of the half-views. Similarly, the other types of stereograms mentioned earlier also consists of half-images in which the perceptual organization of the monocular patterns is quite stable, but here

This research was conducted in the University of Vermont Visual Perception Laboratory and was supported in part by NSF Grant GB-43278 to R.B.L. Requests for reprints should be sent to R. B. Lawson, Psychology Department, John Dewey Hall, University of Vermont, Burlington, Vermont 05401. the laterally displaced portion of the display can be identified in the half-images.

Thus, it appears that the detection of transversely displaced portions of stereoscopic displays requires that the perceptual organization of the monocular patterns be quite stable even if the disparate pattern cannot be detected monocularly. Therefore, we focused our attention upon examining the effects of transversely disparate forms upon stereoscopic depth perception when the monocular patterns yielded reversible and mutually exclusive perceptual organizations. We attempted to generate a visual display in which global disparity could be carried by a labile but monocularly recognizable form that was bounded by subjective contours even in the absence of any binocular disparity. Consequently, we arrived at the configuration shown in Figure 1, which is a modification of the kind of display used frequently to demonstrate principles of perceptual organization and binocular rivalry (Kaufman, 1974, p. 306).

Upon inspection of Figure 1, most observers report that they can see a diamond-like figure appearing in front of a horizontal grid of black and white lines, that the diamond is bound on all four sides by faint edges, and that the white vertical stripes of the diamond appear brighter than the horizontal white stripes. As is evident, there is an alternative perceptual organization that can arise from Figure 1. If you inspect any side of the central diamond, you will see a series of symmetrical partial rectangles, one enclosing another. Here, the subjective contours are eliminated and the entire configuration appears flat and made up of uniformly bright black and white bars. The Figure 1 pattern is different from Kanizsa type configurations in that they contain homogeneous luminance regions bounded by subjective contours and the visual patterns are perceptually stable when viewed monocularly or stereoscopically (Kanizsa, 1955, 1974; Whitmore, Lawson, $\&$ Kozora, 1976). Therefore, the purpose of the 


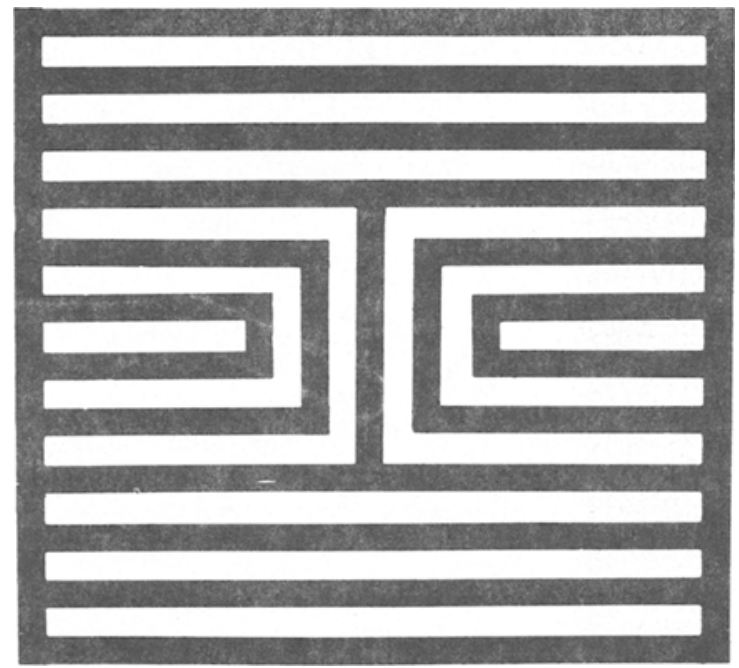

Figure 1. A grid pattern that gives rise to mutually exclusive perceptual organizations of a central diamond or a series of enclosing rectangles. Note that when the diamond appears the corners are blunt or clipped while the sides are bounded by subjective contours.

present experiment was to compare the effects of equivalent disparities carried by patterns that did and did not exhibit perceptual reversibility within the half-images upon the magnitude and duration of stereoscopic depth perception.

\section{METHOD}

\section{Subjects}

Eight females (mean age 24.4 years) served as subjects. Each had normal near visual acuity $(20 / 17-20 / 25)$, normal lateral (3-6) and vertical (6-9) phorias expressed as prism diopter equivalents, and normal stereopsis ( $90 \%$ Fry-Shepard) scores, as measured with a Bausch and Lomb Orthorater (Model 71-21-31). All subjects were naive as to the purpose of this experiment.

\begin{abstract}
Apparatus
Two types of experimental stereograms were employed. In one type, the central diamond was made up of alternating black and white bars, while the other was a solid black diamond (Figure 2). For both types of stereograms, the angular size of the outer rectangular frame was $2.48^{\circ} \times 2.28^{\circ}$, with a side of the central diamond equivalent to $0.67^{\circ}$. For the grid pattern (Figure 2A), the width of the black bars was $0.06^{\circ}$ while the width of the white bars was $0.10^{\circ}$ of visual angle. The luminances of the black and white bars were 1.64 and $30.37 \mathrm{~cd} / \mathrm{m}^{2}$, respectively, under stereoscopic viewing conditions. The disparity carried by the inner diamond varied from $-0.40^{\circ}$ through 0 to $+0.40^{\circ}$ in $0.20^{\circ}$ steps of visual angle. All stereograms were viewed in a prism stereoscope at a distance of $83.5 \mathrm{~cm}$.
\end{abstract}

\section{Procedure}

Each subject was instructed to estimate the stereoscopic distance of the central diamond (grid and solid patterns) or rectangles (grid pattern only) by considering the distance between herself and the outer black rectangular frame as equal to 100 units. Depth estimates of less than 100 indicated that the central diamond or rectangles appeared in front of the rectangular frame while estimates greater than 100 indicated the reverse depth organization.
To estimate the duration of the diamond and rectangular organizations arising from the grid stereograms (Figure 2A). the subject depressed a switch in her right hand that activated a timer (Standard Electric, Model S-1) to indicate the presence of the diamond organization and another switch in the left hand activating a second timer if the rectangular organization was present in the stereoscopic field of view. This response profile was counterbalanced across hands for the eight subjects. Each subject was allowed two trials ( $50 \mathrm{sec}$ duration) to practice the response procedure employing a zero disparate display with a grid pattern orthogonal to the pattern employed for the experimental stereograms.

The solid and grid stereograms were presented twice as counterbalanced blocks across subjects with disparity randomly assigned within blocks. The order of estimating stereoscopic depth and duration of the perceptual organization was counterbalanced across the subjects. The duration of each trial was $50 \mathrm{sec}$, but a depth estimation trial was terminated after the subject reported depth estimates for the diamond (grid and solid patterns) and rectangular organizations (grid pattern only).

\section{RESULTS}

The present results indicate clearly that stereoscopic depth varied directly with disparity regardless of whether the disparity was carried by perceptually reversible or irreversible patterns. Figure 3 presents the mean depth magnitude estimates of the grid and solid diamonds based upon all eight subjects at all disparity levels. Note that there was a slight attenuation of depth magnitude when the disparity was carried by the grid rather than solid patterns regardless of direction of disparity. For the grid pattern, all of the subjects reported depth only when they saw the diamond in the stereoscopic field of view. No depth was reported when the perceptual organization yielded rectangles. Here the subjects reported seeing either the left or right half-view or binocularly overlapping rectangles that appeared coplanar with the remainder of the display. A 2 by 5 by 8 analysis of variance of the mean depth estimates of the central

A
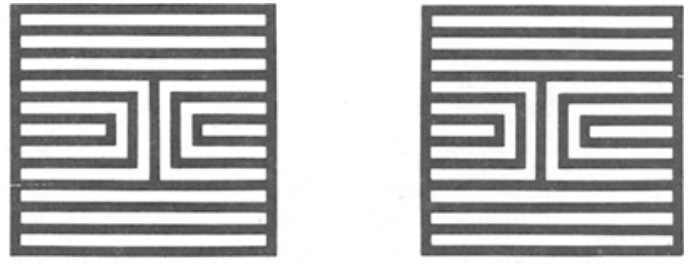

B
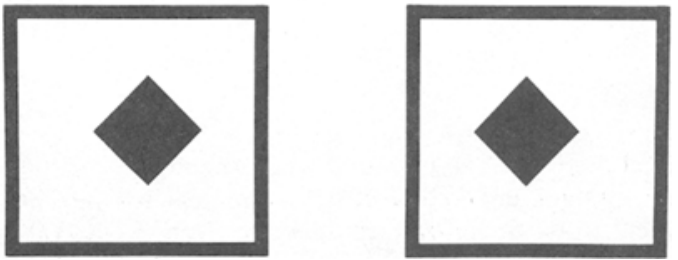

Figure 2. The experimental stereograms containing reversible (A) and irreversible (B) forms. 


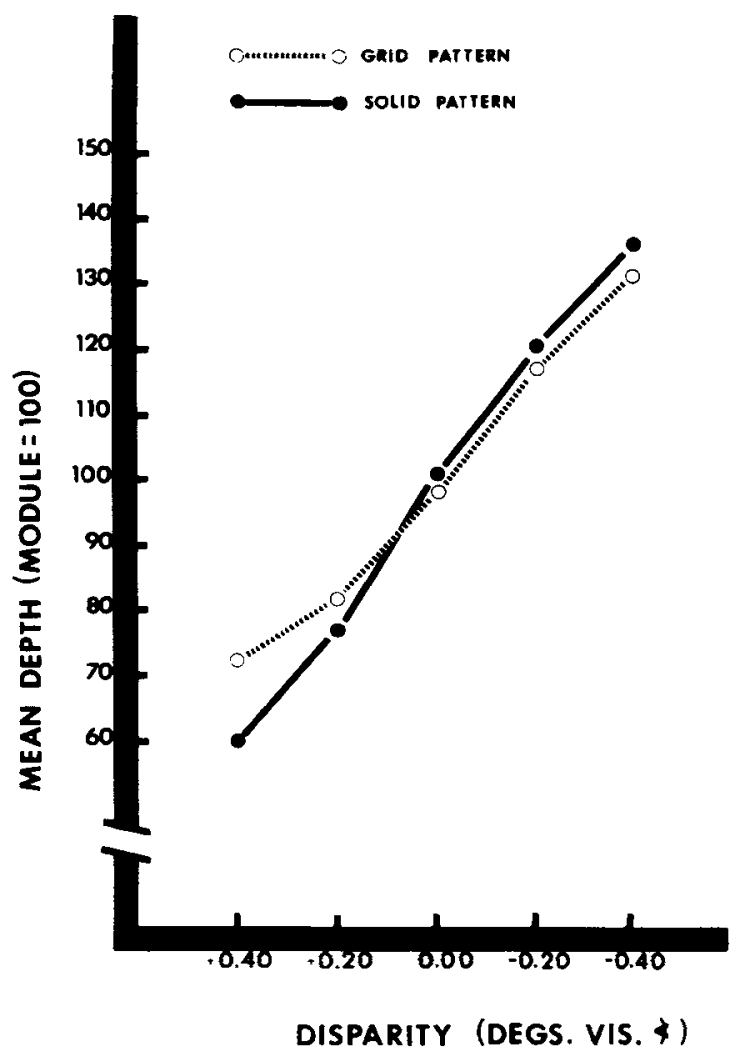

Figure 3. The mean depth estimates based upon all eight subjects for the grid and solid patterns at all five disparity levels.

diamond indicated that the only significant treatment condition was disparity, $F(4 / 28)=21.75, \mathrm{p}<.001$.

For the solid diamond stereograms, all the subjects reported that the stereoscopic organization remained unchanged for the entire period when estimating the duration of the perceptual organization. In terms of the grid stereograms, the present findings also indicate that regardless of disparity level the diamond organization predominated over the rectangular organization. Figure 4 presents the mean duration of the diamond and rectangular perceptual organizations based upon all eight subjects at all five disparities. The subjects reported that the alternations between the perceptual organizations of diamond and rectangles occurred rather abruptly, but once an organization was achieved it remained fairly stable for approximately 5-10 sec. In order to account for elapsed time between perceptual organizations, we subtracted $2.5 \mathrm{sec}$ from the mean estimates of the durations for the rectangular and diamond organizations so that the data points in Figure 4 sum to 45 rather than to the 50 -sec duration of each trial. The crossed or positive disparities not only yielded stereoscopic depth but also stabilized slightly the diamond perceptual organization, while the uncrossed or negative disparities tended to equalize the duration of the rectangular and diamond organi- zations. With crossed disparities, the subjects indicated that the corners of the diamond appeared clearly defined and sharp with all sides of the diamond bounded by crisp subjective contours. With uncrossed disparities, the subjective contours were uncoupled from the more remote diamond and appeared along the inner edges of a central diamond-shaped aperture in the foreground through which the more remote diamond-like target was seen. All observers reported the absence of subjective contours when the rectangular organization arose in the stereoscopic field of view. A 2 by 5 by 8 analysis of variance of the mean duration estimates indicated that the main effect of perceptual organization (diamond vs rectangular organization), $F(1,7)=$ $7.57, \mathrm{p}<.05$, and the Organization by Disparity interaction, $F(4,28)=2.96, p<.05$, were significant.

\section{DISCUSSION}

The present findings indicate clearly that stereoscopic depth perception can arise from perceptually reversible monocular patterns and that the magnitude of reported depth is similar for reversible as well as irreversible disparate patterns. However, compared to irreversible patterns, stereoscopic depth

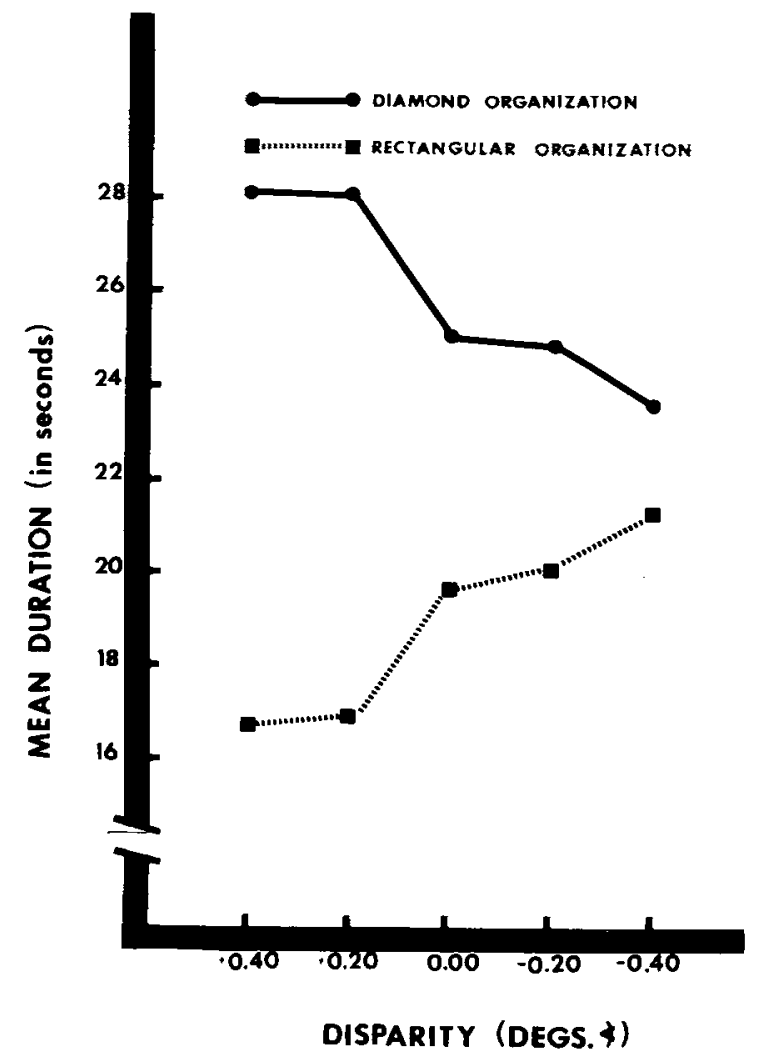

Figure 4. The mean duration of the diamond and rectangular organizations arising from the grid pattern at all five disparity levels. 
magnitude is attenuated slightly regardless of the direction of disparity when carried by reversible monocular patterns.

The results of the present experiment also indicate that the presence of crossed disparity enhances slightly the perceptual stability of the macro-disparate pattern (i.e., the central diamond) but does not preclude perceptual reversibility. However, when the macro-disparate pattern is not visible in the stereoscopic field of view neither stereoscopic depth nor subjective contour perception are reported. Thus, even though human stereopsis can arise in the absence of monocular recognition of the disparate portion of the half-views, failure to stabilize the global form in the combined stereoscopic field, due in the present case to alternating perceptual organizations, eliminates stereoscopic depth perception (Julesz, 1971; Julesz \& Frisby, 1975; Gulick \& Lawson, 1976). It appears that the human binocular system can extract disparity information from monocular patterns exhibiting perceptual alternation, but in order to sustain stereoscopic depth and subjective contour perception the alternation is attenuated at least in the case of crossed or positive disparities, as is evident from Figure 4.

The present findings agree with results from other studies indicating that stereopsis can arise from perceptually labile and spatially disparate half-images (Julesz, 1971; Pandina, Zeller, \& Lawson, Note 1). For example, Julesz (1971) reported that stereopsis arose from the classical "vase-two faces" ambiguous pattern when portrayed in a random-element stereogram. He observed that when the vase was seen closer in stereoscopic space it was bounded by subjective contours and the vase remained as the figure regardless of attempts to shift attention to the more remote faces in order to perceive them as figure. The figure-ground reversals had been eliminated when the pattern was portrayed as a random-element stereogram. However, this was not the case in the present experiment because, even when the diamond organization was seen closer due to crossed disparity, it was still possible to perceive the alternative organization. With the present grid patterns, the perceptual organizations were not limited to a figure-ground reversal, but, rather, involved the perception of mutually exclusive patterns that do not share the same defining contours as is the case for the "vase-two faces" configuration. Thus, when the subjective contours were eliminated in the grid patterns as a result of perceptual alternation, stereopsis was eliminated. Human stereopsis requires contour perception, however the contours defining the disparate portion of the stereoscopic pattern can be generated by retinal or cortical mechanisms.

The present findings make plain that subjective contours can be formed along a surface made up of alternating abrupt luminance gradients as well as against homogeneous luminance regions (Coren, 1972; Kanizsa, 1976; Lawson, Cowan, Gibbs, \& Whitmore, 1974). This result agrees with findings reported by Kennedy (1976) and Gibson (1950), both of whom have presented examples of subjective contours arising along the boundary separating sheared line gratings.

\section{REFERENCE NOTE}

1. Pandina, R., Zeller, P., \& Lawson, R. B. Effects of retinal disparity upon reversible perspective figures. Paper presented at Eastern Psychological Association meetings. New York. 1970.

\section{REFERENCES}

Coren. S. Subjective contours and apparent depth. Psychological Review. 1972, 79, 359-367.

GiBson, J. J. The perception of the visual world. Boston: Houghton Mifflin. 1950

Gulick, W. L., \& Lawson, R. B. Human stereopsis: A psychophysical analysis. New York: Oxford University Press. 1976.

Julesz, B. Foundations of cyclopean perception. Chicago: University of Chicago Press, 1971.

Julesz, B.. \& Frisby, J. P. Some new subjective contours in random-line stereograms. Perception, 1975, 4, 145-150.

Kanizsa, G. Margini quasi-percettivi in campi con stimolazione omogenea. Rivista di psicologia. 1955. 49. 7-30.

Kanizsa. G. Contours without gradients or cognitive contours? Italian Journal of Psychology. 1974, 1. 93-113.

Kanizsa. G. Subjective contours. Scientific American, 1976, 234, 48-52.

Kaufman, L. Sight and mind: An introduction to visual per. ception. New York: Oxford University Press. 1974.

Kaufman, L., \& Pitblado, C. Further observations on the nature of effective binocular disparities. American Journal of Psychology, 1965, 78. 386-389.

KENNEDY, J. M. Attention, brightness and the constructive eye. In M. Henle (Ed.), Vision and artifact. New York: Springer, 1976.

Lawson, R. B., Cowan, E., Gibes, T. D., \& Whitmore, C. G. Stereoscopic enhancement and erasure of subjective contours. Journal of Experimental Psychology, 1974, 103, 1142-1146.

LAWSON. R. B., \& Gurick, W. L. Stereopsis and anomalous contour. Vision Research, 1967, 7, 271-297.

Nelson, J. I. Globality and stereoscopic fusion in binocular vision. Journal of Theoretical Biology, 1975, 49, 1-88.

OGLE, K. N. Theory of stereoscopic vision. In S. Koch (Ed.), Psychology: A study of a science (Vol. 1). New York: McGrawHill, 1959.

Pastore, N. Sebastien Le Clerc on retinal disparity. Journal of the History of the Behavioral Sciences, 1972, 8, 336-339.

Wallach, H., \& BaCon, J. Two forms of retinal disparity. Perception \& Psychophysics, 1976, 19, 375-382.

Wallach, H., \& Lindauer, J. On the definition of retinal disparity. Psychologische Beitrage, 1962, 6, 521-530.

Whitmore, C. L., Lawson, R. B., \& Kozora, C. Subjective contours in stereoscopic space. Perception \& Psychophysics, 1976, 19. 211-213. 